\section{Pneumocephalus following labour epidural analgesia, a rare case} \section{report}

\author{
Shagufta Rafiq ${ }^{*}$ Ibrahim Abbasher, Sadia Anwar, Ammara \\ Ahmad, Bushra Aziz and Branko Denona
}

Department of Obstetrics \& Gynaecology, St. Luke's General Hospital, Kilkenny, Ireland

\section{Abstract}

The lumbar epidural analgesia is commonly used for labour analgesia. The "loss of resistance to air" LORA technique is commonly used for recognition of epidural space. One of the rare complications of this technique is Pneumocephalus (PC). We want to present a case of Pneumocephalus which the mother developed during epidural analgesia in labour. The patient complained of severe headache immediately after attempt at epidural catheter insertion. The symptoms progressively worsened following delivery. A postnatal anaesthetic review was performed and an urgent CT scan of the brain was arranged that showed pneumocephalus. A conservative management pathway was followed with liberal analgesia, oxygen inhalation and keeping the patient mostly in supine position. Her symptoms regressed in severity over the next three days and subsided after one week. We believe that the amount of air used for LORA should be minimized; LORA should not be used after dural puncture and the use of normal saline would alleviate the risk.
More Information

*Address for Correspondence: Shagufta Rafiq, Department of Obstetrics \& Gynaecology, St. Luke's General Hospital, Kilkenny, Ireland, Email:shags_r@msn.com

Submitted: April 22, 2021

Approved: May 04, 2021

Published: May 05, 2021

How to cite this article: Rafiq S, Abbasher I, Anwar S, Ahmad A, Aziz B, et al.

Pneumocephalus following labour epidural analgesia, a rare case report. Clin J Obstet Gynecol. 2021; 4: 066-068.

DOI: 10.29328/journal.cjog.1001089

Copyright: @ 2021 Rafiq S, et al. This is an open access article distributed under the Creative Commons Attribution License, which permits unrestricted use, distribution, and reproduction in any medium, provided the original work is properly cited.

Check for updates

OPEN ACCESS

\section{Background}

The epidural analgesia is commonly used pain relief modality in labouring patients. Pneumocephalus is the air in the intracranial space and it is most commonly encountered after trauma or surgery. It is a rare but serious complication of obstetrical epidural analgesia/anaesthesia. The cranial imaging should be priority even with very low suspicion. PC is relatively common in neurosurgery [1] and neuroradiology $[2,3]$. It can be caused by trauma [4] or infections [5,6]. It may develop after lumbar puncture, epidural steroid injection or valsalva manoeuvre $[7,8]$. The development of PC after spinal or epidural anaesthesia is extremely infrequent. The true incidence of PC after epidural anaesthesia or epidural steroid injection is exactly unknown and only a few cases are described per year in the literature $[9,10]$. The other complications are uncommon and include haemorrhage, CSF leak, PDPH and infection. The treatment of PC is usually conservative but it depends upon the severity of the condition.

\section{Clinical case}

A 21 years old lady, $\mathrm{P} 0+0$ was admitted to the hospital for induction of labour for post-dated pregnancy. Her pregnancy was uneventful and she had regular visits in antenatal clinic. She had a past history of infrequent migraine headaches. The anomaly scan was normal. She was planned for Induction of labour due to post-dated pregnancy as per hospital protocol. Her labour was induced by artificial rupture of membranes followed by oxytocin infusion. Epidural analgesia was given on request when she was in established labour.

The patient was monitored on IMEWS chart and was stable. Two attempts were made at epidural catheter insertion. At first attempt, after locating the epidural space using the LORA technique, the patient complained of severe and sudden headache. Her vital signs remained stable, the needle was withdrawn without further continuation. Subsequently, epidural catheter was cited using the loss of resistance to saline (LORS) technique. The dural tap was suspected by the anaesthetist at the time of first epidural needle insertion trial. She started getting headache immediately after the epidural was cited. Her labour progressed smoothly. She had a forceps delivery with episiotomy due to prolonged second stage of labour. The blood loss during delivery was less than 500 $\mathrm{ml}$. The headache started getting worse half an hour after delivery. She was put on regular analgesia and had a review by the anaesthetic team which advised to continue observation and regular analgesia for 24 hours. The headache was getting worse in severity and it was not affected by any change in position. After adding even multiple potent analgesics, her 
headache was not relieved. She additionally had photophobia, tinnitus, nausea and neck muscle stiffness. There were no focal signs of brain compression. Her movements, gate and reflexes stayed normal. The patient was unable to move her head around and look after the baby. Her CT brain was done next day at review and advice of anaesthetic team and showed two pockets of gas within the left lateral ventricle anteriorly and posteriorly. There was no mass defect seen. She was followed up by the anaesthetic team regularly; neurology team was consulted and was given regular strong analgesia and oxygen inhalation as per advice of neurologist. Close monitoring was continued with conservative management. The severe headache improved gradually over the next few days. She was discharged home on $5^{\text {th }}$ postnatal day in a stable condition with baby (Figures 1,2).

\section{Discussion}

The headache after epidural analgesia is usually ascribed to unintentional dural puncture with consequent CSF leakage through the hole in the dura resulting in low CSF pressure [11]. The development of PC after spinal anaesthesia is exceptionally rare $[12,13]$. This commonly seen headache is called PDPH (Post Dural Puncture Headache) and usually becomes prominent 24 to 48 hours after the dural puncture.

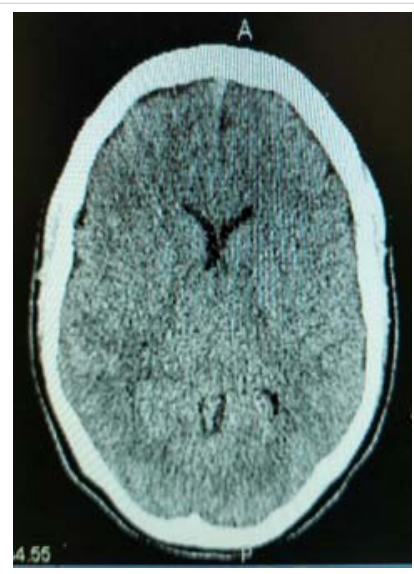

Figure 1: CT scan shows two pockets of gas within the left lateral ventricle anteriorly and posteriorly, no mass defect seen.

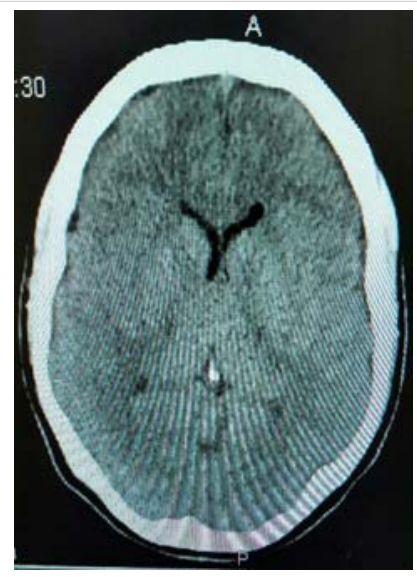

Figure 2: CT scan shows two pockets of gas within the left lateral ventricle anteriorly and posteriorly, no mass defect seen.
The typical symptom is moderate to severe postural headache which is aggravated on standing or sitting. On the other hand, the most common symptom of PC (Pneumocephalus) is severe headache having a sudden onset with gradual improvement over 4-5 days as the accumulated air is resorbed slowly and gradually with the passage of time.

Reynolds and Speedy, [14] suggested that in cases of PDPH without CSF leakage, the sequence of events is as follows: The dura mater is punctured by the Tuohy needle and the delicate arachnoid mater tears leading later to CSF loss and PDPH. In our case, the patient developed moderate headache about 5 minutes after the second epidural catheter insertion attempt without CSF leakage.

There are several ways to avoid pneumocephalus. First, the identification of the epidural space by LOR with saline may be superior to the use of air. Complications related to the saline are rarely reported. LORA, on the other hands has been associated with peumocephalus, nerve root compression, subcutaneous emphysema and venous air embolism [15]. The second preventive measure that practitioners can use in ultrasonography. Spence, et al. [12] provided evidence that pre-procedural ultrasonography is a useful adjunct for the placement of epidural catheters in obstetric patients. If more than a few attempts are required for the epidural placement, ultrasound guidance may be useful to identify the epidural space. Finally, to minimize the possibility of pneumocephalus, when performing labour epidural analgesia with LORA, the minimum amount of air should be used. In addition, if headache develops immediately after the procedure, pneumocephalus should be suspected and CT brain should be performed for confirmation.

Pneumocephalus, a rare consequence of evident or unnoticed accidental dural puncture [14-16], develops from injection of air into the subarachnoid or subdural space and cranial migration. It is not often followed by symptoms, but, when present, headache is the most frequent [17]. The headache is caused by fast brain motion resulting from air injection and meningeal irritation. It is exacerbated by motion and may not be alleviated by lying down [18]. The appearance of other symptoms such as signs of space occupying lesions (focal neurological deficits including cranial nerve palsies $[17,18]$, or diverse motor signs), augmented intracranial pressure and cardiovascular instability may develop depending on the spread and extent of intracranial air. Roderick, et al. Outlined that $2 \mathrm{ml}$ of air injected into the subarachnoid space is sufficient to provoke a symptomatic PC [12].

The treatment of PC consists of administration of 40$100 \%$ oxygen in the supine position. This is to favour the reabsorption of intracranial air by intensifying the diffusion concentration gradient for nitrogen between the air collection and the surrounding cerebral tissue. Nitrous oxide should 
be avoided to prevent the expansion of PC. In addition, we should administer aggressive hydration, caffeine, or analgesia. Epidural infusion or blood patch have no effect on PC [14]. There is usually absorption of air within 3-5 days from the epidural injection and patients commonly improve without any neurological abnormalities. Our patient was discharged as her symptoms regressed drastically after 3 days. If tension PC occurs then neurosurgical emergency treatment may be necessary.

\section{Conclusion}

To conclude, after epidural analgesia; and when particularly dural puncture is performed, the patient should be very carefully monitored. We should identify that headache after PC usually starts immediately after epidural block. In addition, we should recognise that the symptoms are similar to that of PDPH and imaging modalities are necessary to diagnose.

The strategy to minimise this rare but serious complication is to use loss of resistance technique to saline instead of air to identify the epidural space [19]. Every headache that follows epidural analgesia should not be labelled as PDPH. Low threshold should be kept in mind to rule out Pneumocephalus and other pathologies.

\section{Ethical considerations}

Consent was obtained to publish the details of the case, is published anonymously and confidentiality of data storage is assured.

\section{References}

1. Reasoner DK, Todd MM, Scamman FL, Warner DA. The incidence of pneumocephalus after supratentorial craniotomy. Observations on the disappearance of intracranial air. Anesthesiology. 1994; 80: 1008-1012. PubMed: https://pubmed.ncbi.nlm.nih.gov/8017640/

2. Peterson HO, Kieffer SA. Neuroradiology. New York, Hayon and Row. 1984; 1: 127-130.

PubMed: https://www.ncbi.nlm.nih.gov/pmc/articles/PMC7050972/

3. Heinz ER. Techniques in imagimg of the spine, part 3: Myelography, The Clinical Neurosciences: Neuroradiology. Edited by Rosenberg RN New York, Churchill Livingstone. 1984; 795-817.

4. Finch MD, Morgan GA. Traumatic pneumocephalus following head injury. A complication of general anaesthesia. Aneasthesia. 1991; 46: 385-387.

PubMed: https://pubmed.ncbi.nlm.nih.gov/2035788/

5. Candan S, Katelioglu M, Ceylan S, Köksal I. Otogenic brain abcess with pneumocephalus. Infection. 1990; 18: 191-192.

PubMed: https://pubmed.ncbi.nlm.nih.gov/2365472/

6. Finelli P, Bergan R. Spontaneous pneumocephalus with meningitis: CT demonstration. J Comput Assist Tomogr. 1991; 15: 525-526.

PubMed: https://pubmed.ncbi.nlm.nih.gov/2026827/

7. Ozturk E, Kantarci M, Karaman K, Basekim CC, Kizilkaya E. Diffuse pneumocephalus associated with infratentorial and supratentorial haemorrhages as a complication of spinal surgery. Acta Radiol. 2006: 47: 497-500.

PubMed: https://pubmed.ncbi.nlm.nih.gov/16796314/

8. Yoon SJ, Oh GS, Lee SJ, Lee BR, Chun J, et al. Pneumocephalus in patients with orthostatic headache. J Clin Neurol. 2008; 4: 89-93. PubMed: https://pubmed.ncbi.nlm.nih.gov/19513309/

9. Hawley JS, Ney JP, Swanberg MM. Subarachnoid peumocephalus from epidural steroid injection. Headache. 2005; 45: 247-248.

PubMed: https://pubmed.ncbi.nlm.nih.gov/15836602/

10. Simopolulos $T$, Peeters-Asdourian C. Pneumocephalus after cervical epidural steroid injection. Anesth Analg. 2001; 92: 1576-1577. PubMed: https://pubmed.ncbi.nlm.nih.gov/11375849/

11. Han CS, Yu JS, Kim IH, Kim YJ, Kim CS, et al. Headache and pneumocephalus after lumbar epidural block: A case report. J Korean Pain Soc. 1996; 9: 251-255.

12. Spence D, Nations R, Rivera O, Bowdoin S, Hazen B, et al. Evidencebased anesthesia: The use of preprocedural ultrasonography during labor to facilitate placement of an epidural catheter. AANA J. 2012; 80: 223-230.

13. Avellanal M, Olmedilla L, Ojea R, Rueda ML, Navia J. Pneumocephalus after spinal anesthesia. Anesthesiology. 1996; 85: 423-425. PubMed: https://pubmed.ncbi.nlm.nih.gov/8712460/

14. Reynolds F, Speedy HM. The subdural space: the third place to go astray. Anaesthesia. 1990; 45: 120-123. PubMed: https://pubmed.ncbi. nlm.nih.gov/2181886/

15. Kim YJ, Baik HJ, Kim JH, Jun JH. Pneumocephalus developed during epidural anesthesia for combined spinal-epidural anesthesia. Korean $\mathrm{J}$ Pain. 2009; 22: 163-166.

16. Ahlering JR, Brodsky JB. Headache immediately following attempted epidural analgesia in obstetrics. Anesthesiology. 1980; 52: 100-101. PubMed: https://pubmed.ncbi.nlm.nih.gov/7352636/

17. Katz JA, Lukin R, Bridenbaugh PO, Gunzenhauser L. Subdural intracranial air: an unusual cause of headache afterepidural steroid injection. Anesthesiology. 1991; 74: 615-618.

PubMed: https://pubmed.ncbi.nlm.nih.gov/1825771/

18. Nafiu OO, Urquhart JC. Pneumocephalus with headache complicating labour analgesia: should we still be using air? Int J Obstet Anesth. 2006; 15: 237-239.

PubMed: https://pubmed.ncbi.nlm.nih.gov/16798452/

19. Gomez-Rios MA, Fernandez-Goti MC. Pneumocephalus after inadvertent dural puncture during epidural anesthesia; Anesthesiology. 2013; 118: 444.

PubMed: https://pubmed.ncbi.nlm.nih.gov/22692380/ 